\title{
Determination of Small Beam Axial Dose Distribution in Water Based on the Mathematical Model of Pencil Beam Kernels
}

\section{Kolyvanova M.A. ${ }^{1}$, Klimanov V.A. ${ }^{1}$, and Moiseev A.N. ${ }^{2}$}

${ }^{1}$ State Research Center Burnasyan Federal Medical Biophysical Center of the Federal Medical Biological Agency (SRC - Burnasyan FMBC of the FMBA), 46 Zhivopisnaya street, Moscow, 123182 Russia

${ }^{2}$ Ltd MEDSCAN

\section{Abstract}

An analytical model of the dose kernel of pencil beam for photon with the Bremsstrahlung spectrum with maximum energy $6 \mathrm{MV}$ was created in this work, which makes it possible to determine the primary and scattered components of the

Corresponding Author: Kolyvanova M.A.

kolyvanova@physics.msu.ru

Received: 17 January 2018

Accepted: 25 March 2018

Published: 17 April 2018

Publishing services provided by Knowledge $\mathrm{E}$

(c) Kolyvanova M.A. et al. This article is distributed under the terms of the Creative Commons

Attribution License, which

permits unrestricted use and redistribution provided that the original author and source are credited.

Selection and Peer-review under the responsibility of the PhysBioSymp17 Conference Committee. absorbed dose in water phantom with an acceptable error for practice. Using this model a simply method of dosimetry of small circular cross section $6 \mathrm{MV}$ photon beam was proposed. It combines the absolute measurement of the absorbed dose in the water at the reference point for reference geometry with the calculation of the deep dose distribution by simple analytical formulas for circular beams with an arbitrary radius of the cross section.

Keywords: dose kernels, pencil beam, dosimetry, small photon beam, radiotherapy.

\section{Introduction}

In the last two decades widespread medical radiation technology based on creating prescribed dose distributions using superposition of small transverse dimensions fields (IMRT) and precision irradiation of tumors with high doses of beams with small volumes of small circular cross-section diameter of $0.5-3.0 \mathrm{~cm}$ (stereotactic radiotherapy). But there are some problems of small field dosimetry (IMRT, SRS, SBRT):

a) The impossibility of establishing standard reference conditions (reference field size 10x10) for beams calibration in water.

b) Direct measurements in small fields are associated with numerous difficulties: 
- incomplete source visibility from the detection point;

- the effect of averaging over the detector volume;

- perturbations due to the presence of a detector in the radiation field.

These problems led to the development in 2008 of a new formalism to determine the absorbed dose to water in sub-standard conditions [1] taking place in IMRT, radiosurgery and Stereotaxis. Some practical aspects of dosimetry of small fields are considered in the publication IPEM Report 103 [2]. At the same time, while in this area there are many unclear questions that require careful study and detailing [3].

In the present work, it is proposed to use calculated data on deep and radial distributions for circular beams of different sizes, obtained on the basis of pencil beam dose kernels (PB), to improve the accuracy of dosimetry of round small-sized fields. Such data give a relative dependence of different parameters on the radius of the circular field, and in combination with the calibration for MSR-fields - absolute doses values. This approach is seen in the example of a beam of bremsstrahlung $6 \mathrm{MeV}$ photons.

\section{Materials and methods}

Dose kernel of PB (which geometry are introduce on fig.1) is defined as the fraction of PB photons energy absorbed in a unit volume of a semi-infinite medium (in the present work, water) near an arbitrary point $\vec{r}$. Taking into account that in the geometry accepted for the model PB falls normally to a semi-infinite medium, kernels of PB depends on the calculated point depth $z$, its distance from the source axis $r$ and the photon energy $E$. We denote this kernel by $\mathrm{K}_{P B}(E, Z, r)$.
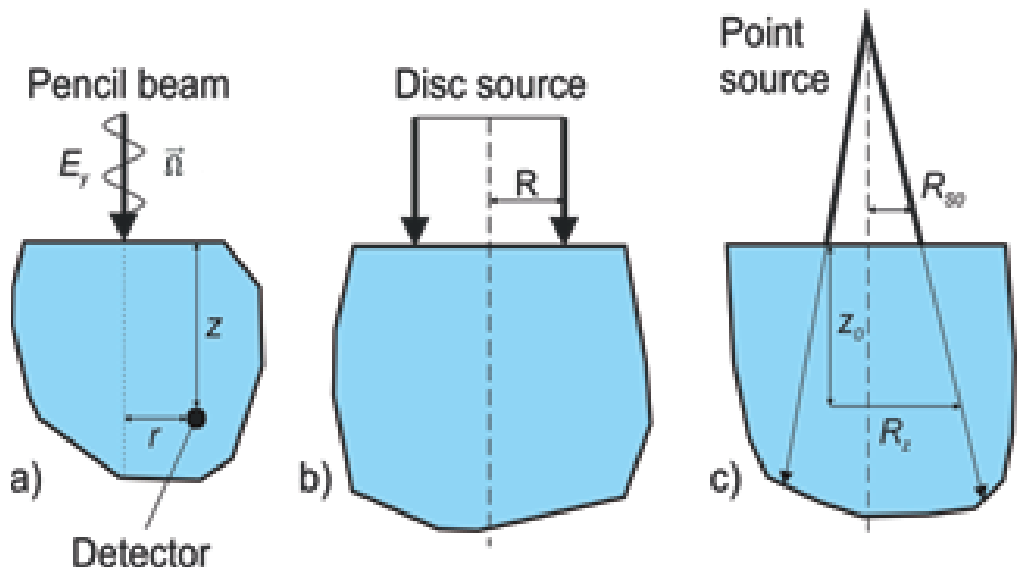

Figure 1: Geometry of a Pencil beam (PB) (a), a disk monodirectional source (b), and a beam with a circular section diverging from point (c). 
Let us analyze the case of a divergent beam (fig. 1c). Since in this paper we study beams with a small circular cross section $(R \leq 3 \mathrm{~cm})$, and taking into account that the distance from the phantom surface to the source is usually $\sim 100 \mathrm{~cm}$, we can take the monodirectional approximation of photons in the beam (the maximum angle of incidence is $\left\langle 1.5^{\circ}\right.$ ). The actual beam divergence will be calculate through the geometric attenuation according to the inverse square law and the change in the size of the integration region $R_{z}$. In this approximation, the dose on the axis of divergent from the point of photon source with radius Rs on the surface and the quality Q (Fig. $1 \mathrm{C}$ ) is determined through the PB dose kernels by the formula:

$$
D\left(Q, z, r=0, R_{s}\right)=2 \pi\left(1+\frac{z}{F}\right)^{-2} \int_{0}^{R} \psi(r, z=0) \cdot \bar{K}_{P B}(z, r) \cdot r \cdot d r
$$

где $R_{z}=(1+z / F) R_{s}, F$ - distance from phantom surface to source.

In this paper, the values $\mathrm{K}_{P B}(E, Z, r)$ in water were determined by the Monte Carlo method for a number of spectra of bremsstrahlung beams from 4 to $24 \mathrm{MV}$ and for the photon spectrum of the therapeutic apparatus ROKUS by the code EGSnrc. In this paper we analyze the results for the $6 \mathrm{MB}$ photon beam, which is most often used in stereotactic irradiation and is presented in the EGSnrc library.

For all spectra of bremsstrahlung photons, PB dose kernels are divided into two components:

$$
K_{P B}=K_{P B, p}+K_{P B, s},
$$

where $K_{P B, p}$ - primary component; $K_{P B, S}$ - scattered component of the dose kernels

For analytical approximation of the radial dependence of each component we used expression (3)

$$
K_{P B, j}(z, r)=\sum_{i=1}^{N} C_{i}(z) \cdot e^{-k_{i}(z) \cdot r} / r,
$$

where $j=\rho$ or $s$ for the primary and scattered components, respectively; $N$ - number of terms in the sum, depending on the beam quality and the type of component; $C_{i}$ and $k_{i}$ - empirical coefficients, which depend on the depth of [4].

For a $6 \mathrm{MB}$ beam, two members in each component were sufficient. The coefficients were found by fitting to the Monte Carlo calculation results. The fitting method described by the authors in [5] was a combination of the random search method and the nonlinear regression method. 
The radial distribution of the energy fluence on the phantom surface can be represented as a homogeneous distribution (4) or described by a polynomial expression or by the sum of the exponential terms (5)

$$
\begin{gathered}
\Psi(r, z=0)=\left\{\begin{array}{l}
\text { const for } r \leq R_{s} ; \\
0 \text { for } r>R_{s},
\end{array}\right. \\
\Psi(r, z=0)=\left\{\begin{array}{l}
\sum_{n=0}^{N} a_{n} \cdot r^{n}, \text { for } r \leq R_{s} ; \\
\sum_{n=0}^{N} b_{n} \cdot e^{c_{n} r}, \text { for } r>R_{s},
\end{array}\right.
\end{gathered}
$$

For a beam with a circular cross section diverging from the point

$$
D(Q, z, r=0)=2 \pi \cdot \text { const } \cdot\left(1+\frac{z}{F}\right)^{-2}\left[\frac{C_{1, j}}{k_{1, j}}\left(1-e^{-k_{1, j}\left(1+\frac{z}{F}\right) R_{s}}\right)+\frac{C_{2, j}}{k_{2, j}}\left(1-e^{-k_{2, j}\left(1+\frac{z}{F}\right) R_{s}}\right)\right] .
$$

The integral (1) are also taken analytically.

Dependence of the dose component on the dose value in the reference point with a radius $\mathrm{R}_{0}$ (for CyberKnife $R_{0}=3 \mathrm{~cm}$ ) at a depth $\mathrm{z}_{0}$ :

$$
D_{j}\left(Q, R_{s}, z, 0\right)=D_{j}\left(Q, R_{S_{0}}, z_{0}, 0\right)\left(\frac{g_{0}}{g}\right)^{2} \frac{\left[\frac{C_{1, j}(z)}{k_{1, j}(z)}\left(1-e^{-k_{1, j}(z) R_{z}}\right)+\frac{C_{2, j}(z)}{k_{2, j}(z)}\left(1-e^{-k_{2, j}(z) R_{z}}\right)\right]}{\left[\frac{C_{1, j}\left(z_{0}\right)}{k_{1, j}\left(z_{0}\right)}\left(1-e^{-k_{1, j}\left(z_{0}\right) R_{z_{0}}}\right)+\frac{C_{2, j}\left(z_{0}\right)}{k_{2, j}\left(z_{0}\right)}\left(1-e^{-k_{2, j}\left(z_{0}\right) R_{z_{0}}}\right)\right]},
$$

где $g_{0}=\left(1+Z_{0} / F\right) ; g=(1+Z / F) ; R_{z}=g R_{s} ; R_{z_{0}}=g_{0} R_{s_{o}}$.

The total dose in both cases, according to the model, is equal to the sum of the two components. We introduce two functions for compactness of the final equation:

$$
\begin{aligned}
& f_{p}\left(C_{1}, C_{2}, k_{1}, k_{2}, g, z, R_{s}\right)=\frac{C_{1, p}(z)}{k_{1, p}(z)}\left(1-e^{-k_{1, p}(z) g R_{s}}\right)+\frac{C_{2, p}(z)}{k_{2, p}(z)}\left(1-e^{-k_{2, p}(z) g R_{s}}\right) ; \\
& f_{s}\left(C_{1}, C_{2}, k_{1}, k_{2}, g, z, R_{s}\right)=\frac{C_{1, s}(z)}{k_{1, s}(z)}\left(1-e^{-k_{1, s}(z) g R_{s}}\right)+\frac{C_{2, s}(z)}{k_{2, s}(z)}\left(1-e^{-k_{2, s}(z) g R_{s}}\right) .
\end{aligned}
$$

The expression for the Total Dose:

$$
\begin{aligned}
D\left(Q, R_{s}, z, r=0\right)=D\left(Q, R_{s_{0}}, z_{0}, r=0\right)\left(\frac{g_{0}}{g}\right)^{2} \times \\
\times\left[\frac{f_{p}\left(C_{1}, C_{2}, k_{1}, k_{2}, g, z, R_{s}\right)+f_{s}\left(C_{1}, C_{2}, k_{1}, k_{2}, g, z, R_{s}\right)}{f_{p}\left(C_{1}, C_{2}, k_{1}, k_{2}, g_{0}, z_{0}, R_{s_{0}}\right)+f_{s}\left(C_{1}, C_{2}, k_{1}, k_{2}, g_{0}, z_{0}, R_{s_{0}}\right)}\right] .
\end{aligned}
$$




\section{Results}

Calculations of the dose nucleus of TL photons for the $6 \mathrm{MW}$ bremsstrahlung were carried out according to the code EGSnrc separately for each component for $z=0.25$ $39.75 \mathrm{~cm}$ in $0.5 \mathrm{~cm}$ steps and for $r \leq 46 \mathrm{~cm}$ until a relative statistical error $\delta<1 \%$. The step of energy losses by charged particles was $2 \%$. Based on the results obtained, the mathematical analytical model described above was constructed. The relative meansquare error of calculating results $<5 \%$.

To test the proposed method of dosimetry of circular small-sized beams, a point on the depth dependence of the absorbed dose in water was chosen as the reference "calibration" point for $z=4,75 \mathrm{~cm}$ and $r=0$ for $R_{s}=3,0 \mathrm{~cm}$. The determination of the dose at this point was carried out using the dose kernels of PB by formula (1), assuming a homogeneous distribution of energy fluence in the cross section at the phantom surface. According to the formula (9) below, the depth dose distributions for circular cross section divergent beams with different radius on the water phantom surface were determined for $R_{s}=0,25 ; 0,5 ; 1,0$ and $3,0 \mathrm{~cm}$ at $F=100 \mathrm{~cm}$. These distributions are compared in Fig. 2 with the results of calculating the doses in these geometries by the Monte Carlo method using two methods.

The ones shown in Fig. 2 comparisons show that in both cases a good coincidence of the results is observed.
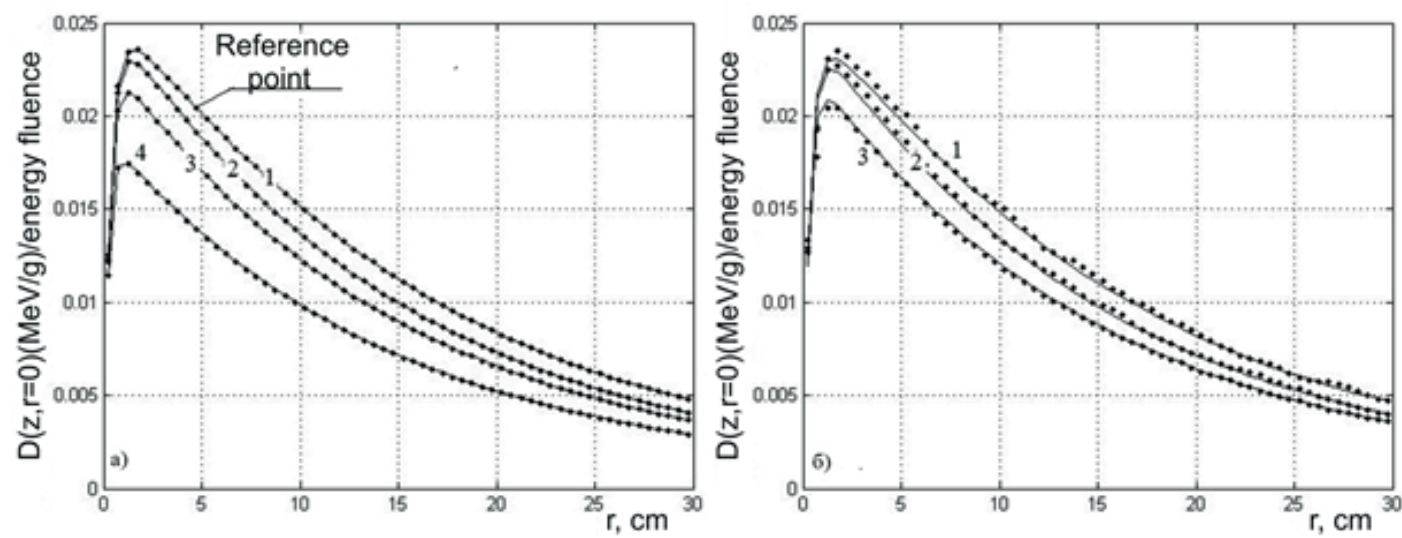

Figure 2: Comparison of depth dose distributions obtained by the proposed method (formula 8) and formed by divergent beams of circular cross section in the water phantom with (a) the results of the calculation by a combination of Monte Carlo method and the PB method, (b) with the results of direct Monte Carlo method (EGSnrs code) for different beams radii on the surface. The dose on the beam axis with a radius of $3 \mathrm{~cm}$ at a depth of $4.75 \mathrm{~cm}$ was taken as the reference dose. Designations: $1-\mathrm{R}_{s}=3 \mathrm{~cm}$; $2-\mathrm{R}_{s}=1.0 \mathrm{~cm} ; 3-\mathrm{R}_{s}=0.5 \mathrm{~cm} ; 4-\mathrm{R}_{s}=0.25 \mathrm{~cm}$. 


\section{Conclusion}

As a result of the research, a mathematical model of Pencil Beam dose kernels for photons with a $6 \mathrm{MV}$ bremsstrahlung spectrum was developed, including a convenient analytical approximation of the radial dependence of the dose kernels and the value of the empirical coefficients included in the analytic approximation. The error in calculating the PB dose kernels for the proposed model is less than $5 \%$. A mathematical model of the dose kernels formed the basis for the method of dosimetry beams with a small cross section $(\mathrm{R}<3 \mathrm{~cm})$, combining the absolute measurement of dose in a reference point in a machine-specific geometry and analytical calculation depth dose distributions for simple formulas for circular beams.

\section{References}

[1] R. Alfonso, P. Andreo, R. Capote et al. A new formalism for reference dosimetry of small and nonstandard fields, Med. Phys., vol. 35, № 11. pp. 5179-5186, 2008.

[2] IPEM. Small field MV photon dosimetry. Technical Report 103. Institute of Physics in Engineering and Medicine, York, 2010.

[3] H. Bouchard, J. Seuntjens, S. Duane, et al., Detector dose response in megavoltage small photon beams. I. Theoretical concepts, Med. Phys., vol. 42, № 10, pp. 60336047, 2015.

[4] P. Kazantcev, V. Klimanov, Application of the ionization method in the dosimetry of small photon therapeutic beams, Medical physics, vol. 47, № 3, pp. 14-22, 2010.

[5] V. Klimanov, A. Moiseev, M. Kolyvanova et al., The dose kernels of pencil and differential pencil photon beams with the spectrum of treatment machines with a 60Co source in water and their analytical approximation, Moscow University Physics Bulletin, vol. 71, №4, pp. 432-439, 2016. 